\title{
Synthesis and Structural Analysis of an H-Shaped Polybutadiene
}

\author{
Sébastien Perny and J urgen Allgaier* \\ Institut für Festkörperforschung, Forschungszentrum J ülich, 52425 J ülich, Germany
}

\section{Donghyun Cho, Wonmok Lee, and Taihyun Chang*}

Department of Chemistry and Center for Integrated Molecular Systems, Pohang University of Science and Technology, Pohang, 790-784, Korea

Received September 18, 2000

\begin{abstract}
The synthesis of an $\mathrm{H}$-shaped polybutadiene homopolymer as well as its detailed structural characterization is investigated. Anionic polymerization techniques together with chlorosilane linking agents were used for the production of the material. After each reaction step samples were taken and analyzed by size exclusi on chromatography (SEC), membrane osmometry (MO), NMR, and temperature gradient interaction chromatography (TGIC). According to the characterization by SEC, MO, and NMR, the $\mathrm{H}$-polymer showed a high degree of structural uniformity after purification by fractionation, and no significant amounts of differently branched byproducts could be detected. The TGIC analysis however revealed the presence of large amounts of structures, mainly with lower branching degree. We also found that a significant isotope effect exists in the TGIC retention between deuterated and hydrogenous polymers. In our case, where the H-polymer is partially deuterated, the TGIC analysis enabled us to resolve all the side products. Comparison of the different analysis methods indicates that the precise structural analysis of branched model polymers such as the $\mathrm{H}$-polymer requires more sophisticated methods than used in the past.
\end{abstract}

\section{Introduction}

Three-arm star polymers represent the simplest example for branched polymers. The next complex structure is the $\mathrm{H}$-polymer, where two sidearms are attached to the backbone chain. These polymers play an important role as model materials in rheology, for example to understand the processing behavior of branched polymers such as LDPE. ${ }^{1-3}$

In recent years several publications appeared reporting the synthesis of $\mathrm{H}$-polymers or super $\mathrm{H}$-polymers. In the latter case three arms are attached to each end of the cross-bar chain instead of two as for the $\mathrm{H}$-shaped polymers. ${ }^{4-9}$ The synthetic procedures, however, are similar for both structures. Anionic polymerization techniques were used together with chlorosilanes or aromatic diolefins as coupling agents. Most of those materials were made as block copolymers using different monomers or different microstructure for the cross-bar and the arms. By this means the reactivity of the living chain ends could be adjusted as required by the corresponding reaction steps in order to ease the reaction or to suppress the appearance of side products.

The characterization of model polymers usually is based on size exclusion chromatography (SEC), assisted by membrane osmometry (MO), light scattering (LS), and NMR. Agreement of the characterization measurements with the expected results is normally used to conclude that the materials represent model polymers of high structural homogeneity. Characterization of branched structures like star polymers or $\mathrm{H}$-polymers by SEC, however, is difficult. SEC separates polymer molecules according to their volume in solution, which changes very little with changing branching degree. In this case a different chromatographic technique, temperature gradient interaction chromatography (TGIC), has proven its usefulness. TGIC is an interaction chromatography technique. Separation of polymer mol- ecules is driven by enthal pic interactions of the solute molecules with the stationary phase, and the interaction strength is varied by changing the column temperature during the elution. ${ }^{10,11}$ These interactions are in a first approximation proportional to the molecular weight and independent of the polymer volume. As already demonstrated with linear materials, the resolution power of TGIC is far better than SEC; ${ }^{12-14} \mathrm{TGIC}$ is an ideal tool for the structural analysis of branched materials, as was demonstrated successfully with a 6-arm star polystyrene. 15,16

This paper describes the synthesis of a polybutadiene (PB) $\mathrm{H}$-shaped homopolymer. The material was synthesized for small-angle neutron scattering (SANS) investigations. To perform SANS experiments, the crossbar was produced with deuterated monomer. The product was characterized by SEC, MO, NMR, and TGIC.

\section{Experimental Section}

Materials. Cyclohexane, n-hexane, benzene (Merck, >99\%), and triethylamine (TEA) (Fluka, 99.5\%) were purified by distillation from solvent freen-butyllithium. TEA was left over n-butyllithium no longer than $15 \mathrm{~h}$ before it was distilled off. Butadiene (Aldrich, 99\%) and deuterated (d) butadiene (Chemotrade, 98 atom \% d) were purified by treatment with solvent free dibutylmagnesium overnight at $0{ }^{\circ} \mathrm{C}$, then distilled onto solvent free n-butyllithium, and allowed to stand for 20 min at $-20^{\circ} \mathrm{C}$ immediately before use or transfer into ampules equipped with a break-seal. 1,1-Diphenylethylene (DPE) (Aldrich, 97\%) was purified by treatment with solvent-free n-butyllithium, distilled under high-vacuum conditions, and diluted in benzene. Finally, the DPE solution was subdivided into ampules. As the weights of DPE and benzene were measured, it was possible to determine the concentration of the solution precisely.

Methyltrichlorosilane (Fluka, 99\%) was first stirred over $\mathrm{CaH}_{2}$ overnight and then purified by distillation on the vacuum line. The first third of the distillate was discharged, the next third collected, diluted with cycl ohexane, and transferred into 
ampules. The concentration was determined by hydrolyzing an aliquot with water and titrating the $\mathrm{HCl}$ formed with standard sodium hydroxide solution.

sec-Butyllithium (Aldrich) was distilled under dynamic high vacuum, then diluted with cyclohexane to obtain a concentration of approximately $0.1 \mathrm{~mol} / \mathrm{L}$, and subdivided into ampules. The difunctional initiator was obtained by contacting 1,2-bis[4-(1-phenyl ethenyl)phenyl] ethane (PEPE) with sec-butylithium. PEPE was a gift from C. Fernyhough (University of Sheffield/UK). It was produced according to literature procedures ${ }^{17,18}$ and was freeze-dried with benzene under high vacuum conditions several times. The difunctional initiator was obtained by reacting sec-butyllithium with PEPE in a 2:1 molar ratio in an all-glass apparatus. A mixture of $60 \mathrm{vol} \%$ n-hexane and 40 vol \% benzene was used as solvent. Within $10 \mathrm{~min}$ a deeply red color developed, and after $1 \mathrm{~h}$ insoluble dilithium adduct started to precipitate. After 3 days the product was filtered via a glass filter attached to the apparatus, washed several times with new n-hexane, redissolved in benzene, and finally subdivided into ampules. The concentrations of sec-butyllithium and the difunctional initiator were determined by hydrolyzing an aliquot with water and titrating the $\mathrm{LiOH}$ formed with standard $\mathrm{HCl}$ solution.

Polymerizations. The manipulations were performed under high vacuum in all-glass reactors, provided with break seals for the addition of reagents. A mixture of $85 \mathrm{vol} \%$ of dry benzene and $15 \mathrm{vol} \%$ of TEA was used as solvent for the synthesis. The arm synthesis was performed by first distilling $56.7 \mathrm{~g}(1.05 \mathrm{~mol})$ of hydrogenous (h) butadiene and then 130 $\mathrm{mL}$ of TEA and $720 \mathrm{~mL}$ of benzene into the reactor, which was cooled with liquid nitrogen. The reactor was sealed off, and the ampule containing $2.24 \mathrm{mmol}$ of sec-butyllithium was opened. After $20 \mathrm{~h}$ a sample was taken, by transferring $24 \%$ of the solution into an ampule attached to the reactor. The reactor and the ampule were cooled with a dry ice bath, and the ampule was sealed off. The polymer was terminated with degassed methanol in a drybox, precipitated in methanol, and dried in a vacuum oven.

The difunctional cross-bar was synthesized separately using $22.1 \mathrm{~g}(0.37 \mathrm{~mol})$ of d butadiene, $47 \mathrm{~mL}$ of TEA, and $190 \mathrm{~mL}$ of benzene. $0.40 \mathrm{mmol}$ of the 2:1 adduct of sec-butyllithium and PEPE in $70 \mathrm{~mL}$ of benzene was used as initiator. After all ingredients except the initiator were mixed in the reactor, $2-3 \%$ of the overall initiator amount was added. This measure should neutralize the remaining protic impurities, which cause monofunctional living chains. A strong yellow color indicated the absence of impurities, and therefore the rest of the initiator was added in one batch. After 3 days 3\% of the polymer was removed for the cross-bar analysis. The living chain ends were capped with an equimolar amount of DPE for 6 days, and a red col or developed. During this time three $1 \mathrm{~mL}$ samples were taken to monitor the capping reaction. Then the solution was mixed quickly with $6.24 \mathrm{~g}(41.7 \mathrm{mmol})$ of $\mathrm{CH}_{3} \mathrm{SiCl}_{3}$. Within a few minutes the solution became colorless. The mixture was left overnight, the excess $\mathrm{CH}_{3} \mathrm{SiCl}_{3}$ and the solvent mixture were distilled off at the vacuum line into a different flask, and the material was left under dynamic vacuum overnight. The process was repeated two times by first redissolving the solid polymer in $80 \mathrm{~mL}$ of dry benzene. Finally, the polymer was dissolved in $160 \mathrm{~mL}$ of dry benzene, and the reactor was sealed off at dry ice temperature. $45 \mathrm{~mL}$ of the solution was transferred into an ampule attached to the reactor and seal ed off as described before. This material was used for other experiments. The reactors containing the rest of the cross-bar solution and the living arm material were combined and mixed whereby the excess of living chain ends over $\mathrm{Si}-\mathrm{Cl}$ groups was approximately $50 \%$. Initially the color of the mixture was yellow due to the living arm chain ends. Within 6 days it turned to orange, most likely because of aggregation of the chain ends with the $\mathrm{LiCl}$ formed. The color stayed constant until after 3 weeks the reaction was terminated with methanol, and the $\mathrm{H}$-polymer separated in a series of fractionation cycles with toluene as solvent and methanol as nonsolvent to remove excess arm material as well as low and high molecular weight byproducts. First the polymer was dissolved in toluene, and then methanol was added slowly until the solution became slightly turbid. In the next step more methanol was added, and the solution was heated until it became dear. The solution was transferred into a separation funnel and left overnight in order to cool to room temperature. After separation of the two layers, the polymer fractions were analyzed by SEC. In a typical experiment $0.3 \mathrm{~L}$ of methanol was needed per $1 \mathrm{~L}$ of toluene, and the polymer concentration was approximately $1 \%$ in the first fractionations and then lowered to approximately $0.25 \%$ in the last cycles. If low molecular weight material was removed, the quantity of excess methanol was in the range that the solution became clear $8-9^{\circ} \mathrm{C}$ over room temperature, and the lower fraction was used for the next separation step. For the removal of high molecular weight material the excess methanol quantity was smaller so that the solution became clear $2{ }^{\circ} \mathrm{C}$ over room temperature and the upper fraction was taken. The whole procedure was repeated until the shape of the SEC trace remained constant. Finally, from $57 \mathrm{~g}$ of polymer mixture $19 \mathrm{~g}$ of product could be isolated.

Measurements. SEC experiments were carried out at 30 ${ }^{\circ} \mathrm{C}$, using a Waters $150 \mathrm{C}$ instrument together with four Styragel columns with a porosity range from $10^{5}$ to $500 \AA$, a differential refractometer, and a UV detector. The solvent was THF, and the flow rate was $1 \mathrm{~mL} / \mathrm{min}$. PS standards (Tosoh Corp.) were used for the calibration. The end-capping of the living PB cross-bar with DPE was monitored by SEC using UV detection. During the reaction samples were taken, terminated with methanol, and the solvent was removed completely from the samples at room temperature under vacuum. High boiling DPE did not evaporate under these conditions. The weight of the polymer-DPE mixture was measured, and the mixture was redissolved in a known amount of THF. The DPE signal intensity was compared with calibration measurements obtained from samples of known DPE concentrations. Knowing the sample weight, the polymerDPE weight ratio and finally the end-capping degree could be calculated.

Number-average molecular weights $M_{n}$ were measured with a Knauer membrane osmometer at $37^{\circ} \mathrm{C}$. The solvent was toluene, which was distilled from sodium. The results were obtained from the square root plot $(\pi / \mathrm{C})^{1 / 2}=(\pi / \mathrm{c})_{\mathrm{c}=0^{1 / 2}}(1+$ $0.5 \mathrm{M}_{n} \mathrm{~A}_{2} \mathrm{C}$ ), where $\pi$ is the osmotic pressure and $\mathrm{A}_{2}$ is the second virial coefficient. ${ }^{19}$

Microstructures and deuterium contents were measured by $\mathrm{NMR}$ in $\mathrm{CDCl}_{3}$ using a $300 \mathrm{MHz}$ Bruker spectrometer. ${ }^{1} \mathrm{H}$ NMR was used to determine the microstructure of the hydrogenous components, and ${ }^{13} \mathrm{C} N \mathrm{NMR}$ was used for the deuterium cross-bar. The deuterium content of the $\mathrm{H}$-polymer was determined by ${ }^{1} \mathrm{H}$ NMR, examining known amounts of polymer together with the ref 1,1,2,2-tetrabromoethane. The deuterium content was calculated from the signal intensity ratios.

IC experiments were carried out on a typical HPLC system equipped with a C18 bonded silica column (Alltech, Nucleosil, $100 \AA$ pore, $250 \times 4.6 \mathrm{~mm}, 5 \mu \mathrm{m}$ particle size). Mobile phase was 1,4-dioxane (HPLC grade, Fisher Scientific) at a flow rate of $0.5 \mathrm{~mL} / \mathrm{min}$. The polymer samples were dissolved in a portion of the elution solvent at a concentration of $6 \mathrm{mg} / \mathrm{mL}$. This concentration is somewhat high for typical analyses but necessary for a reasonable signal/noise ratio for light scattering detection. The effect of concentration on resolution was checked using a much lower concentration (1-2 mg/mL). For most cases, it does affect the retention time to some extent but does not seriously change the resolution. The sample solution was injected through a six-port injector (Rheodyne 7125) equipped with a $50 \mu \mathrm{L}$ sample loop. The column temperature was controlled in a preprogrammed manner by circulating water from a bath/circulator (N eslab, RTE-111) through a homemade column jacket. Chromatograms were recorded with a combination of the foll owing detectors: a refractive index (RI) detector (Shodex RI-71), an evaporative light scattering detector (ELSD, Polymer Lab.), and a low-angle laser light scattering (LS) detector $\left(7^{\circ}, 633 \mathrm{~nm}\right)$ (LDC Analytical, KMX-6). Although it is not suitable for quantitative measurements, ELSD detection provides the best baseline stability as well as sensitivity for 
Scheme 1. Synthesis of H-Polymers
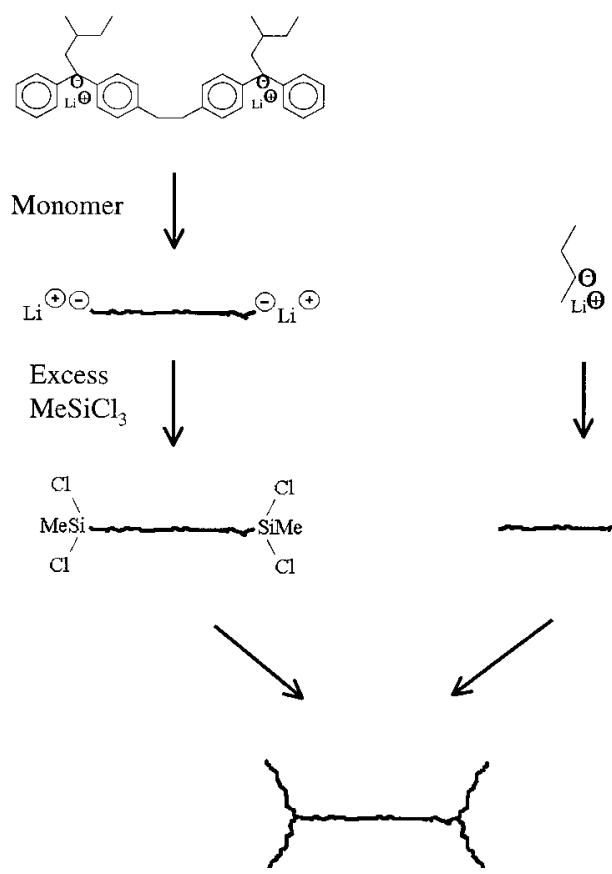

polymer samples. Ther efore, ELSD detection was used when a quantitative analysis was not necessary. LS detection was used in conjunction with a concentration detector when information on absolute molecular weights was required.

To obtain precise values, the refractive index increments, $\mathrm{dn} / \mathrm{dc}$, were determined separately with a Chromatix KMX 16 differential refractometer at $633 \mathrm{~nm}$. The partially deuterated $\mathrm{H}$-polymers represent block copolymers with respect to their isotope composition. The $\mathrm{dn} / \mathrm{dc}$ values of $\mathrm{d}$ and $\mathrm{h}$ polymers are different, and the $\mathrm{d} / \mathrm{h}$ composition of the structures changes with the cross-bar/arm ratio if differently branched byproducts are taken in account. Therefore, $\mathrm{dn} / \mathrm{dc}$ was measured for $\mathrm{d}$ and h polymers having a degree of $42-44 \%$ of 1,2 microstructure in the TGIC solvent 1,4-dioxane at $20^{\circ} \mathrm{C}$. The following values were found: $h$ PB, 0.0967 ; $\mathrm{PB}, 0.0845$. These values are al ready corrected for $100 \%$ isotope purity. Since the relationship between $\mathrm{dn} / \mathrm{dc}$ and $\mathrm{d} / \mathrm{h}$ composition is linear for blocky structures, dn/dc can be calculated for each possible branched structure.

\section{Results and Discussion}

1. Synthesis and Characterization by SE C, MO, and NMR. The synthetic pathway used to produce the $\mathrm{H}$-polymer is given in Scheme 1. First the difunctional cross-bar was synthesized by anionic polymerization in an all-glass reactor and functionalized with methyltrichlorosilane. In the second step, the monofunctional arms were produced and then coupled with the crossbars. The polymers were synthesized in a mixture of benzene and TEA. Polar cosolvents are usually not used for polydiene model polymer syntheses as they might induce side reactions due to the higher reactivity of the chain ends compared to purely nonpolar solvents. TEA however, is a relatively mild additive and was needed in our case to obtain approximately $40 \%$ of 1,2 -microstructure in the polymer. This increased the $T_{g}$ of the product to $-60^{\circ} \mathrm{C}$, which was necessary for the physical investigations.

Cross-Bar. The adduct of PEPE and sec-butyllithium is a hydrocarbon-soluble difunctional initiator. In nonpolar solvents this initiator leads to broad and bimodal molecular weight distributions due to slow initiation. As TEA was used as cosolvent, these difficulties did not
Table 1. Characterization of the H-Polymer

\begin{tabular}{lccccl}
\hline & $\begin{array}{c}\mathrm{M}_{\mathrm{n}} \\
\text { (MO) }\end{array}$ & $\begin{array}{c}\mathrm{M}_{\mathrm{n}} \\
\text { calcda }^{\mathrm{c}}\end{array}$ & $\begin{array}{c}\mathrm{M}_{\mathrm{w}} / \mathrm{M}_{\mathrm{n}} \\
\text { (SEC) }\end{array}$ & $\begin{array}{c}\text { \% 1,4-microstr } \\
\text { (NMR) }\end{array}$ & $\begin{array}{c}\text { isotope } \\
\text { label }\end{array}$ \\
\hline cross-bar & 57200 & & 1.04 & 58 & $\mathrm{~d}$ \\
arm & 25000 & & 1.02 & 56 & $\mathrm{~h}$ \\
H-polymer & 161000 & 157000 & 1.04 & & $\mathrm{~d} / \mathrm{h}$
\end{tabular}

a Calculated from $\mathrm{M}_{\mathrm{n}}$ cross-bar and $\mathrm{M}_{\mathrm{n}}$ arm.

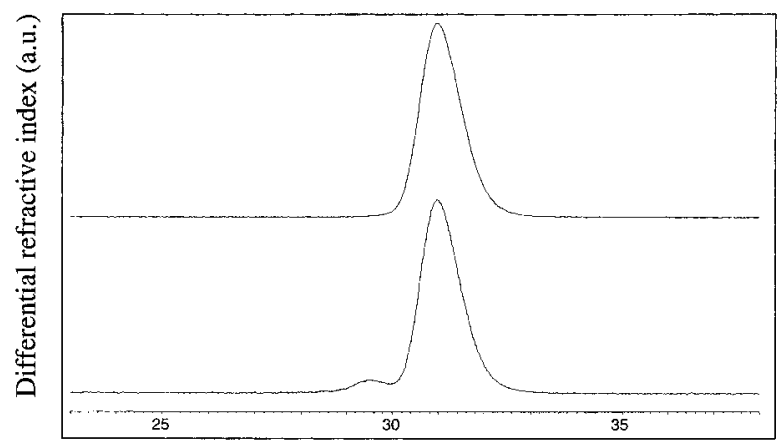

Elution volume $(\mathrm{mL})$

Figure 1. SEC traces of the cross-bar before (upper part) and after functionalization (lower part) with methyltrichlorosilane.

exist. The polydispersity of the cross-bar is in the range expected for monofunctional initiators, and the SEC trace is almost symmetric in shape (see Table 1 and Figure 1). This indicates that the quality of the difunctional initiator was good. In the next step, the carbonlithium chain ends were functionalized with methyltrichlorosilane. The strategy was to use a 25 -fold molar excess of the silane compound over carbon-lithium chain ends to suppress the condensation of two crossbar chains via the same chlorosilane molecule. On the other hand, too large of an excess of silane should be avoided because of the possible presence of $\mathrm{HCl}$ impurities in the chlorosilane which terminate carbon-lithium chain ends. This protonation reaction is faster than coupling of the chain ends to the silicon center. For that reason, the reactivity of the $\mathrm{PB}$ chain ends was reduced by end-capping with DPE prior to the silane functionalization.

DPE does not homopolymerize anionically. ${ }^{20,21}$ It was added only in a 3\% molar excess to the chain ends because unreacted DPE cannot be eliminated by evaporation due to the high boiling point. However, it would end-cap the living polymer arms in the next step and reduce their reactivity. After the addition of the calculated amount of DPE to the cross-bar solution the color change from light yellow (PB chain ends) to dark red (DPE capped chain ends) took many hours. Although TEA was present, the sl ow color change indicated a low reactivity of the end-capping event.

To ensure complete end capping, samples were taken during the reaction and analyzed by SEC using UV detection. Typical SEC traces of a partially end-capped polymer are shown in Figure 2. The upper chromatogram was obtained by UV detection. The signal at 48.4 $\mathrm{mL}$ corresponds to DPE; the cross-bar signal appears at $31.2 \mathrm{~mL}$. Because of its extended delocalized structure, unreacted DPE has a higher extinction coefficient in the UV light than DPE attached to the chain ends. For that reason, even at high end-capping rates the DPE signal intensity is much stronger than the polymer signal intensity. By comparing the signal intensities of the samples with calibration measurements, it was possible to calculate the end-capping degrees. The 


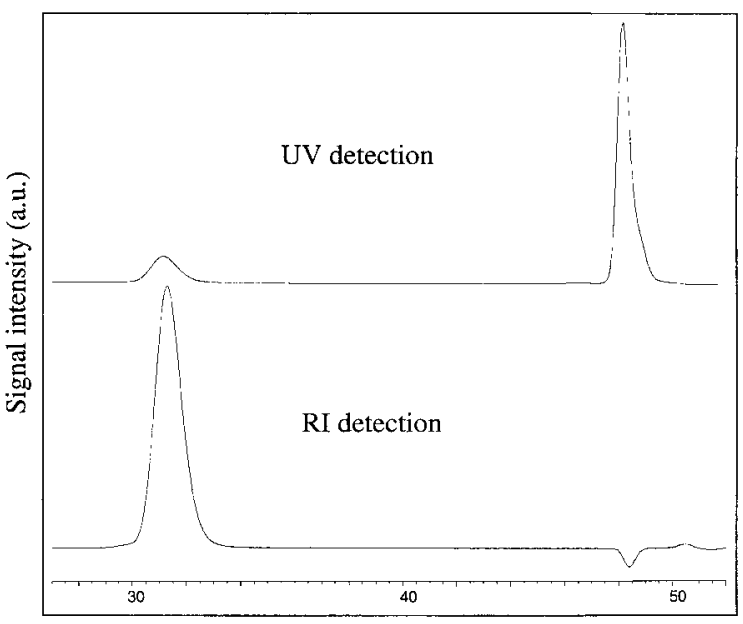

Elution volume $(\mathrm{mL})$

Figure 2. SEC traces of living PB capped with DPE after 40 $\mathrm{h}$ of reaction time

Table 2. End-Capping of Living PB with DPE

\begin{tabular}{cccc}
\hline $\begin{array}{r}\text { reaction } \\
\text { time }(\mathrm{h})\end{array}$ & $\begin{array}{c}\text { DPE concn in } \\
\text { SEC sample } \\
(\mu \mathrm{mol} / \mathrm{L})\end{array}$ & $\begin{array}{c}\text { overall amount of DPE } \\
\text { in polymerization } \\
\text { reactor }(\mathrm{mmol})\end{array}$ & $\begin{array}{c}\text { end-capping } \\
\text { deg }(\%)\end{array}$ \\
\hline 0 & & 0.80 & 0 \\
20 & 89.5 & 0.46 & 44 \\
40 & 54.6 & 0.34 & 60 \\
140 & 5.1 & 0.02 & 101
\end{tabular}

method is described in detail in the Experimental Section. F or thetrace shown in Figure 2 the end-capping degree was $60 \%$. The sensitivity of the UV detection is underlined if we compare the UV detection with RI detection (lower trace in Figure 2). In the latter case the DPE signal is so small that it is hidden by the negative solvent peak appearing at slightly higher elution time. The values collected in Table 2 also document how sensitive the UV method is to detect even smallest amounts of DPE. Finally, the results show that it took between 40 and $140 \mathrm{~h}$ until all PB chain ends were capped and that almost no unreacted DPE was left.

As mentioned before, the living polymer chain ends were functionalized with an excess of methyltrichlorosilane. Unreacted silane was distilled off under vacuum, and a sample was taken for SEC analysis (see lower part in Figure 1). Although the PB chain ends were capped with the highly bulky DPE, the small signal at lower elution volume indicates approximately $5 \%$ of coupled product. Principally, the coupling of two crossbar chains also could take place outside the reactor by reaction of two $\mathrm{Si}-\mathrm{Cl}$ groups with a water molecule, forming a $\mathrm{Si}-\mathrm{O}-\mathrm{Si}$ bond. This reaction can be excluded under the conditions used in this work. ${ }^{22}$

Arms. The H-polymer arms were synthesized with the same microstructure as the corresponding cross-bars, using sec-butyllithium as initiator. The characterization is given in Table 1.

$\mathrm{H}$-Polymer. $\mathrm{H}$-polymers were obtained by reacting the $\mathrm{Si}-\mathrm{Cl}$ functional ized cross-bar chains with a $50 \%$ excess of living arm polymer. Samples were taken after 1 and 3 weeks of reaction time and analyzed by SEC. In all cases the signal shape remained unchanged with time and the intensity ratios of arm signal to product signal stayed constant. For that reason complete linking was assumed, and the reaction was quenched with methanol after 3 weeks.

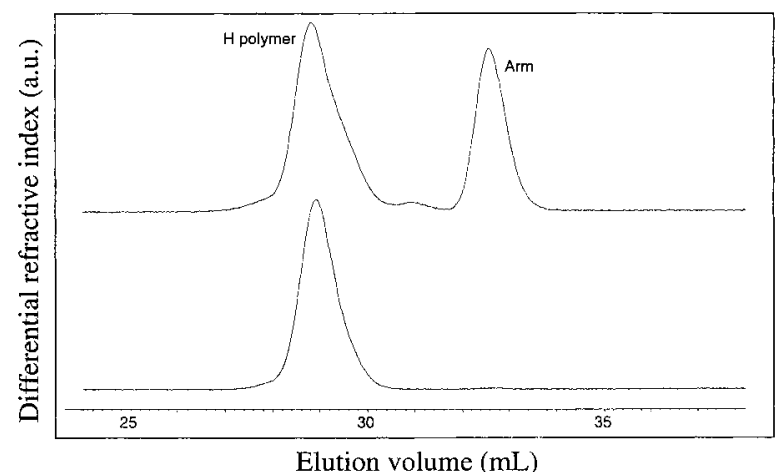

Figure 3. H-polymer before (upper part) and after fractionation (lower part).

The SEC trace of the unfractionated raw product in Figure 3 shows the $\mathrm{H}$-polymer together with the excess of unreacted arm material. The inspection of the $\mathrm{H}$ signal reveals the presence of lower and higher branched structures. The small shoulder at the high molecular weight side of the $\mathrm{H}$ signal shows the presence of structures with two cross-bars and up to five arms. This finding is in agreement with the cross-bar analysis, where coupled product was found after reaction with methyltrichlorosilane. The large excess of chlorosilane over carbon lithium units and the end-capping of the chain ends with DPE should prevent chain coupling. It is known that DPE end-capped living polymers replace only one chlorine atom quickly when reacted with silicon tetrachloride. Replacement of the second chlorine atom takes weeks even in the presence of small amounts of THF ${ }^{23}$ However, it is possible that the high amount of TEA increased the reactivity so much that some coupling could take place. Because of the high reactivity of the system, it also was not possible to first couple two living sidearms with a chlorosilane unit and then add the cross-bar. This pathway would suppress highly branched product and was successfully used in the first report about $\mathrm{H}$-polymer synthesis by Roovers and Toporowski. ${ }^{24}$

The asymmetry of the $\mathrm{H}$ signal and the small signal between the $\mathrm{H}$ and the arm signal in the raw product SEC trace indicates the presence of structures where only three or less arms are attached to the cross-bar. The possible side products are shown in Scheme 2.

The raw material was fractionated to eliminate both high and low molecular weight side products along with excess arm material. Details are given in the Experimental Section. The fractionation procedure was stopped when the shape of the $\mathrm{H}$-polymer signal in the SEC trace remained constant. This criterion does not necessarily mean that the product was free of side products, which have molecular weights not too different from that one of the $\mathrm{H}$-polymer and therefore are difficult to eliminate by fractionation. The SEC signal of the product (lower part in Figure 3) shows a small tailing on the left side which can be due to high molecular weight side products. Additionally, the shape of the signal is not fully symmetric because of a small skewing at the low molecular weight side. As the skewing is only a little different from that one of the cross-bar (see Figure 1), it remains unclear whether low molecular weight side products are responsible or whether the skewing is a rudiment of the slightly unsymmetrical cross-bar distribution. The molecular weight characterization is in good agreement with the expected values 
Scheme 2. Differently Branched Byproducts during the Formation of H-Polymers

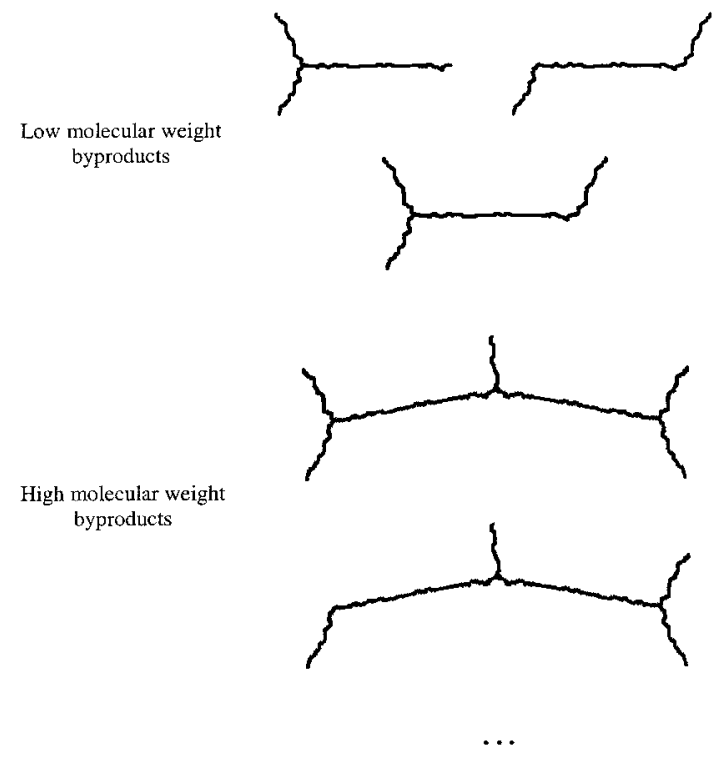

(Table 1). As the H-polymer was produced with a completely deuterated cross-bar and completely hydrogenous arms, the $\mathrm{d} / \mathrm{h}$ compositi on also yields information about the structural quality of the product. The $d / h$ composition was measured by ${ }^{1} \mathrm{H}$ NMR, as described in the Experimental Section. Considering that d PB contains $0.8 \%$ of hydrogen, the $h$ content in the pure $\mathrm{H}$-polymer should be $63.8 \%$. The measured value is $62.8 \%$, which agrees with the expected result and supports the molecular weight characterization.

If we recapitulate the results obtained from the characterization by SEC, MO, and NMR, we conclude that the product represents the expected $\mathrm{H}$-polymer and is uniform in structure. Small amounts of byproduct with different branching degree certainly cannot be detected by the methods used, and their presence cannot be excluded. Moreover, our findings are in good agreement with the other reports dealing with the synthesis of branched model polymers.

2. Characterization by TGIC. The resolution power of TGIC is generally superior to SEC. Additionally, SEC resolves branched polymers worse than their linear counterparts of the same mol ecular weights. Here again TGIC is advantageous as its resolution power is less sensitive to the molecular architecture. ${ }^{15,16}$ For these reasons we decided to subject the $\mathrm{H}$-polymer to a more detailed inspection by TGIC.

The Isotope Effect in TGIC. The molecular weight separation in TGIC results from enthal pic interactions of the polymer chains with the solvent and the stationary phase. Therefore, the method is restricted to polymers with a uniform composition. In our case the $\mathrm{H}$-polymer was produced as homopolymer with respect to the chemical composition, but concerning isotope labeling it represents a block copolymer.

Normally, h and d molecules behave almost identical with respect to their chemical behavior and most physical properties. However, isotopic labeling affects the retention in interaction chromatography, in particular in reversed phase chromatography. 25-27 Since TGIC is an interaction chromatography technique and a reversed phase stationary phase is used, the isotope effect is expected.
In the first series a pair of linear $h$ and $d$ 1,4-PB was investigated. According to MO measurements, both polymers have the same chain length within experimental error and molecular weights in the range of $150000 \mathrm{~g} / \mathrm{mol}$. The SEC and TGIC results are shown in Figure 4. As expected, the SEC elution times are identical. The scenario changes dramatically for the TGIC measurements. The retention time for the $d P B$ is much smaller than for the $h$ PB.

However, at this stage we are not able to clearly attribute the differences between $h$ and $d$ PB to an isotope effect. Small differences in microstructure or different polymerizable impurities in the $\mathrm{h}$ and $\mathrm{d}$ monomer also could be responsible for the differences. To check this, the low molecular weight substances n-decane and n-dodecane were analyzed. Figure 4 also shows the results for $n$-dodecane- $d_{26}$ and $n$-dodecane$\mathrm{h}_{26}$. The scenario is the same as for PB. A fraction of the dodecane molecules containing a chemical impurity would generate additional peaks at different retention times, which is not the case. For that reason, a microstructure effect or different polymerizable impurities in the $d$ and $\mathrm{h}$ monomers can be excluded, and the isotope effect must be responsible for the different retention times of $h$ and $d$ materials. The isotope effect appears to increase with molecular weight. At a first view, the quantity of the effect is astonishing. On the other hand, $h$ and $d$ materials show a small but significant difference in polarizability expressed in $\mathrm{dn} / \mathrm{dc}$ values, which are $10-25 \%$ higher for the $h$ versions. This just highlights how sensitive TGIC is against small changes of the enthalpic interactions between polymer, solvent, and stationary phase. To our knowledge such an isotope effect has not been reported in the chromatographic separation of polymers so far. The isotope effect is also sensitive to the choice of the stationary phase, and we did not find a measurable effect in the normal phase (bare silica) TGIC.

The consequences of the isotope effect on the analysis of the H-polymer are as follows. Because of the changing cross-bar to arm ratio for the different structures, the $\mathrm{d} / \mathrm{h}$ ratio is also different for each structure. From the details listed in Table 1 it is possible to calculate the expected d content for each structure. These values are collected in Table 3 together with the corresponding $\mathrm{dn} / \mathrm{dc}$ values. Principally, the higher the d content of a structure is, the earlier it will be eluted compared to its fully hydrogenous counterpart. This fact can lead to advantages for the TGIC analysis of the $\mathrm{H}$-polymer in this study as will be discussed in the next section.

Inspection of the $\mathrm{H}$-Polymer. Figure 5 displays five TGIC chromatograms of the different synthesis stages recorded by a RI detector: from the top, $\operatorname{arm}(A)$, crossbar (B), raw product (C), fractionated product (D), and a high molecular weight fraction $(E)$. The temperature of the circulating fluid was changed linearly during the elution as shown in the upper abscissa of the plot. The negative peaks appearing near $4 \mathrm{~mL}$ of elution volume for all the chromatograms are the injection solvent peaks. The molecular weight dependence on elution time is opposite to SE C because of the different fractionation principles. Therefore, the higher molecular weight polymers elute at longer retention times. However, the cross-bar (B) elutes earlier than arms (A) although the molecular weight of the cross-bars is twice as large as the arms. This indicates that the higher molecular weight of the cross-bar is slightly overcompensated by 

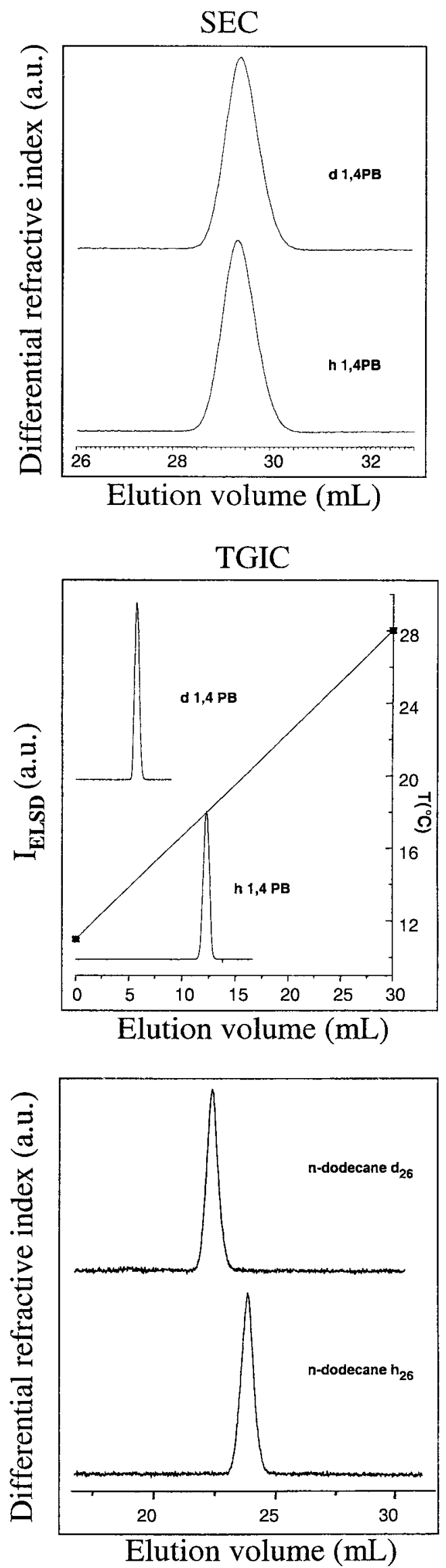

Figure 4. TGIC and SEC analysis of deuterated materials.

the isotope effect. The shift of the cross-bar peak makes a large retention gap between the cross-bars and $\mathrm{H}$ -

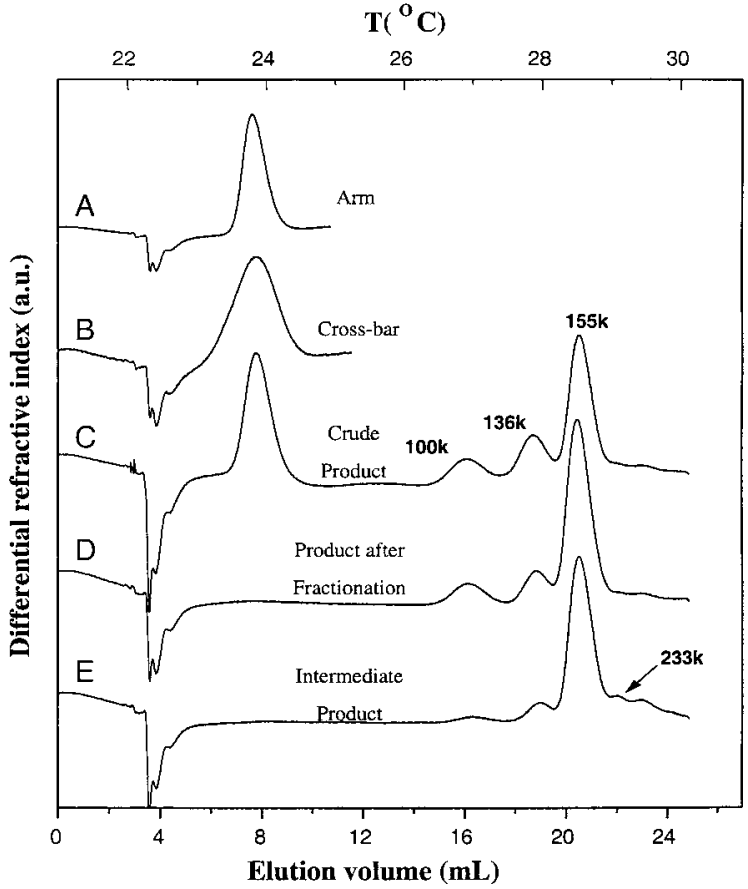

Figure 5. TGIC analysis of different stages during the $\mathrm{H}$-polymer synthesis.

Table 3. Mass Fraction of Deuterated Monomer Incorporated into Different Structures and $\mathrm{dn} / \mathrm{dc}$ Values in 1,4-Dioxane at $20^{\circ} \mathrm{C}$ and $633 \mathrm{~nm}$

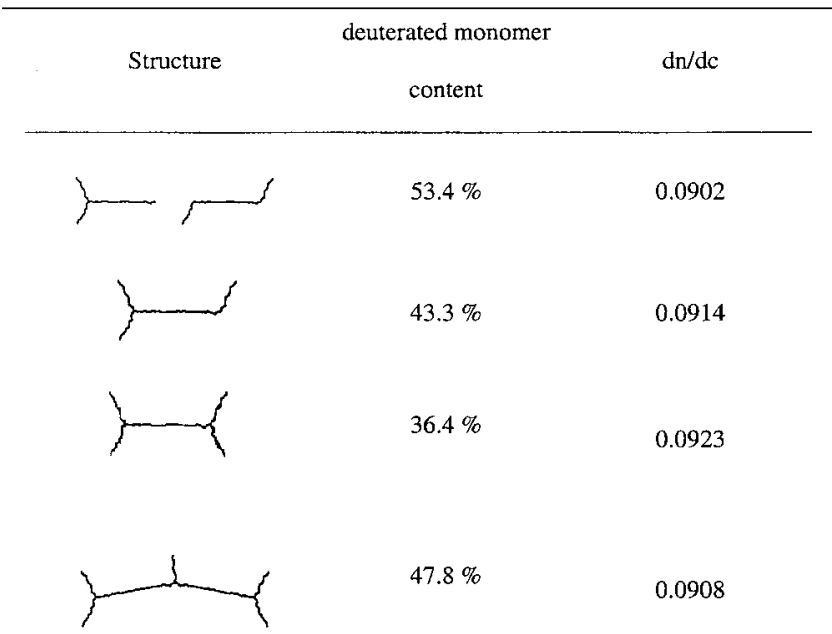

polymers, which enhances the resolution of the intermediate peaks in between. In the chromatogram of the crude product (C), several elution peaks are visible. Beside residual arm at early el ution, the product is split up into three conspicuous peaks and shoulders on the high molecular weight side. In agreement with the linking chemistry, they should represent the $\mathrm{H}$-polymer and byproducts of different branching degree shown in Scheme 2. After fractionating in order to remove low and high molecular weight impurities, the chromatogram of the final $\mathrm{H}$-polymer product (D) shows no remaining arm material. However, the product still contains a substantial amount of byproducts. The relative amounts of low and high molecular weight impurities are smaller than in the unfractionated sample but still significant. In addition to $70 \%$ of $\mathrm{H}$-polymer, TGIC analysis revealed $25 \%$ of lower branching degree material and $5 \%$ of higher branched products. Compared to the SEC results, these findings are impressive. In the SEC trace of the crude product, differently branched 
byproducts were visible; however, the purified product looked clean.

For a more detailed analysis, online LS detection was used to obtain the peak molecular weights of each signal. The determination of the molecular weights needed an approximation. Since there is $1-2 \%$ difference in $\mathrm{dn} / \mathrm{dc}$ between the different structures as shown in Table 3, the concentration measurement from the RI detector signal intensity is not straightforward. However, we assumed identical dn/dc for the determination of the concentration because this assumption should not affect the results significantly. As the peaks are not fully resolved, we determined the peak molecular weights and not average molecular weights, which is again sufficient to identify the structure of the peaks. On the other hand, we used the dn/dc values shown in Table 3 to calculate the molecular weight from the scattering intensity and the concentration since it involves (dn/ dc) ${ }^{2}$, and it is possible to assign $\mathrm{dn} / \mathrm{dc}$ for each peak with a priori assumption of the structure. The calculated molecular weights are shown in Figure 5. They correspond well with the calculated values for the $\mathrm{H}$ polymer (157K), the structure where one arm is missing (132K), and the structure with two missing arms (107K). These results also agree with the structural assumptions based on the linking chemistry.

Additional samples representing different stages during fractionation were examined. They had similar TGIC traces with variation of the relative amounts of low and high molecular weight components. The chromatogram $\mathrm{E}$ in Figure 5 was obtained from one of these samples, where high molecular weights were enriched. At the right side of the $\mathrm{H}$-polymer signal a few clear peaks were obtained. The peak molecular weight of the first signal was determined as $233 \mathrm{~K}$. This value agrees with the calculated molecular weight of the structure consisting of two cross-bars and five arms of $239 \mathrm{~K}$ and confirms the former assumptions.

We now have to analyze the role of the isotope effect. Because of the use of $\mathrm{d}$ cross-bar and $\mathrm{h}$ arm, as the number of missing arms increases, molecular weight decreases while d content increases (see Table 3). These two effects both increase the retention gap between the peaks of the differently branched polymers due to missing arms, i.e., increases resolution, and the peaks of the polymers are better separated from each other. On the other hand, higher branched structures are less separated from the H-polymer. However, comparison of the high molecular weight sides of TGIC and SEC clearly shows that the resolution power of TGIC is still better. The difference in molecular weight between $\mathrm{H}$-polymer and the structure where one arm is missing is only $16 \%$. Without isotope labeling, we cannot expect such a good peak separation from this small molecular weight difference due to the finite molecular weight distribution even for a well made polymers by anionic polymerization. Nevertheless, due to the isotope effect in combination with the high resolution power of TGIC, it is possible to fully resolve the different species in this particular model polymer.

How can such a high amount of byproducts be explained from the synthetic standpoint? The formation of higher branched structures is due to cross-bar condensation and was discussed earlier. The presence of such structures at least in the crude product was al ready clear from the SEC examination of the $\mathrm{Si}-\mathrm{Cl}$ functionalized cross-bar. In the case of the lower branched structures the linking reaction was not complete. We found that a reaction time of 1 week was sufficient for complete linking to prepare three-arm stars with similar molecular weights, ${ }^{28}$ whereas the $\mathrm{H}$-polymer reacted 3 weeks. Therefore, steric hindrance may play a role. The DPE-capped cross-bar chain ends are extremely bulky. If the PB chain ends were linked to the silicon center to some extent in the 1,2 sense, which also is relatively bulky, the second arm may not couple to the silicon center. This explanation is supported by another experiment, in which we attempted to synthesize a four-arm star using $\mathrm{SiCl}_{4}$ and 1,2 PBLi in a polar environment. The product consisted of a mixture of three- and fourarm stars, and the completeness of the linking reaction did not change with increasing reaction time. In the case of the $\mathrm{H}$-polymer the solution was orange, indicating living arm chain ends, until the linking reaction was stopped. We can therefore exclude early termi nation of the chain ends.

The relatively high amount of structures containing only two arms could be explained differently. If the batch of methyltrichlorosilane used for the functionalization of the living cross-bar chains contained some $\mathrm{HCl}$, this would lead to termination of chain ends and finally to a fraction of three-arm star material in the product.

The question now arises as to why conventional analysis using SEC, MO, and NMR leads to a very different picture about the structural purity of the $\mathrm{H}$-polymer. To understand the discrepancies, we have to critically review the first analysis results. SEC separates polymer chains by their size in solution. In branched structures this quantity changes little with different branching degrees. For that reason, careful inspection of the signal shape allows to detect relatively high amounts of impurities in the raw product. Smaller amounts of impurities in the product remain undetected due to the insensivity of the method. MO and NMR are also relatively insensitive for this purpose because of the foll lowing considerations. U sing the measured crossbar and arm molecular weights, the calculated $M_{n}$ of the pure $\mathrm{H}$-structure is $157000 \mathrm{~g} / \mathrm{mol}$ and the calculated $M_{n}$ of the product composition obtained from TGIC is $151000 \mathrm{~g} / \mathrm{mol}$. The product molecular weight from the MO measurement is $161000 \mathrm{~g} / \mathrm{mol}$. The error bar for such a measurement is in the range 5-10\%. If we now compare the different values, it is understandable that the MO measurements cannot yield precise information about the structural homogeneity of the $\mathrm{H}$-polymer. For the NMR measurements the results are similar. Pure $\mathrm{H}$-polymer should have a calculated h content of $63.8 \%$ and the product mixture according to the TGIC measurement $60.6 \%$. The NMR measurement itself yields $62.8 \%$. If we assume an error bar of $5 \%$ for the measurement, it is obvious that NMR also cannot exclude the presence of even large amounts of impurities.

\section{Conclusion}

Characterization of branched model polymers such as star polymers or $\mathrm{H}$-polymers is conventionally performed by a combination of SEC and MO, LS, and NMR. High structural uniformity of the product is concluded from symmetric and narrow SEC signals and the agreement of the MO, LS, and NMR results with the expected values. For our investigation of an $\mathrm{H}$-shaped polybutadiene we used TGIC as an additional analysis technique. Comparison of the conventional analysis by 
SEC and MO and NMR with TGIC reveals substantial discrepancies. Conventional analysis suggests that the product is of high structural quality after fractional purification. However, the TGIC investigation revealed large amounts of differently branched byproducts in the $\mathrm{H}$-polymer. This is because the resolution power of TGIC is far superior to SEC, and especially for branched polymers, the reliability of SEC is low. Without the isotope effect, however, separation of the polymers with different branching degree was not possible even with the high resolution of TGIC. Additionally, simple calculations also show that even large amounts of differently branched side products change the average values for the product $\mathrm{M}_{\mathrm{n}}$ or composition only in a range that is still covered by the error bar of the analysis method. We therefore need to be more alert of the fact that precise structural analysis of branched model polymers such as the $\mathrm{H}$-polymer is not possible with conventional analysis techniques but requires more sophisticated methods than used in the past.

Acknowledgment. S.P. and J .A. thank the European Union for funding this work under the Industrial \& Materials Technol ogies Program (Brite Euram). T.C. acknowledges support from KRF (BK21 Project) and from KOSEF (Center for Integrated Molecular Systems). The authors are grateful to R. N. Young and C. Fernyhough (University of Sheffield) for providing samples of diolefin, L. Willner (J ülich) for his help in molecular weight characterization, and R. Zorn for discussing the isotope effect.

\section{References and Notes}

(1) Ball, R. C.; McLeish, T. C. B. Macromolecules 1989, 22, 1911.

(2) McLeish, T. C. B.; Allgaier, J .; Bick, D. K.; Bishko, G.; Biswas, P.; Blackwell, R.; Blottière, B.; Clarke, N.; Gibbs, B.; Groves, D. J .; Hakiki, A.; Heenan, R. K.; J ohnson, J. M.; Kant, R.; Read, D. J .; Young, R. N. Macromolecules 1999, 32, 6734.

(3) Utracki, L. Adv. Polym. Technol. 1985, 41.

(4) Hakiki, A.; Young, R. N.; McLeish, T. C. B. Macromolecules 1996, 29, 3639.

(5) Gido, S. P.; Lee, C.; Pochan, D. J .; Pispas, S.; Mays, J. W.;
Hadjichristidis, N. Macromolecules 1996, 29, 7022.

(6) I atrou, H.; Avgeropoulos, A.; Hadjichristidis, N. Macromolecules 1994, 27, 6232

(7) Archer, L. A.; Varshney, S. K. Macromol ecules 1998, 31, 6348.

(8) Hadjichristidis, N.; Xenidou, M.; I atrou, H.; Pitsikalis, M.; Poulos, Y.; Avgeropoulos, A.; Sioula, S.; Paraskeva, S.; Velis, G.; Lohse, D. J .; Schulz, D. N.; Fetters, L. J .; Wright, P. J Mendelson, R. A.; Garcia-Franco, C. A.; Sun, T.; Ruff, C. J Macromol ecules 2000, 33, 2424.

(9) Paraskeva, S.; Hadjichristidis, N. J. Polym. Sci., Polym. Chem. Ed. 2000, 38, 931.

(10) Lee, H. C.; Chang, T. Polymer 1996, 37, 5747.

(11) Chang, T.; Lee, H. C.; Lee, W.; Park, S.; Ko, C. Macromol. Chem. Phys. 1999, 200, 2188.

(12) Lee, W.; Lee, H. C.; Chang, T.; Kim, S. B. Macromolecules 1998, 31, 344.

(13) Lee, W.; Lee, H. C.; Park, T.; Chang, T.; Chae, K. H. Macromol. Chem. Phys. 2000, 201, 320.

(14) Lee, W.; Lee, H. C.; Cha, J .; Chang, T.; Hanley, K. J .; Lodge, T. P. Macromolecules 2000, 33, 5111.

(15) Lee, H. C.; Chang, T.; Harville, S.; Mays, J. W. Macromolecules 1998, 31, 690.

(16) Lee, H. C.; Lee, W.; Chang, T.; Yoon, J . S.; Frater, D. J .; Mays, J. W. Macromol ecules 1998, 31, 4114.

(17) I kker, A.; Möller, M. New Polym. Mater. 1993, 4, 35.

(18) Lattermann, G.; Höcker, H. Makromol. Chem. 1974, 175, 2865.

(19) Mays, J. W.; Hadjichristidis, N. Measurement of Molecular Weights of Polymers by Osmometry. In Modern Methods of Polymer Characterization, Barth, H. G.; Mays, J. W., Eds.; J ohn Wiley \& Sons: New York, 1991

(20) Evans, A. G.; George, D. B. J . Chem. Soc. 1961, 4653.

(21) Evans, A. G.; George, D. B. J . Chem. Soc. 1962, 141

(22) Frater, D. J .; Mays, J . W.; J ackson, C.; Sioula, S.; Efstradiadis, V.; Hadjichristidis, N. J . Polym. Sci., Polym. Phys. Ed. 1997, 35, 587

(23) Allgaier, J .; Young, R. N.; Efstratiadis, V.; Hadjichristidis, N. Macromolecules 1996, 29, 1794.

(24) Roovers, J .; Toporowski, P. M. Macromolecules 1981, 14, 1174.

(25) Klein, P. D. Adv. Chromatogr. 1996, 3, 3.

(26) Tanaka, N.; Thornton, E. R. J . Am. Chem. Soc. 1976, 98, 1617.

(27) Sampson, R. L. In Liquid Chromatography of Polymers and Related Materials; Cazes, J., Ed.; Dekker: New York, 1976; p 149.

(28) Own unpublished results and L. J. Fetters, private communication.

MA001608V 